\title{
Responsabilidad social corporativa: de concepto abstracto a elemento clave en la estrategia empresarial
}

\author{
Corporate social responsibility: \\ of an abstract concept to a key \\ element in the business strategy
}

\author{
Begoña Gómez Nieto (España) \\ gomeznietob@gmail.com \\ Universidad de Segovia \\ Doctora en Ciencias de la Información
}

\author{
Rocío Martínez Domínguez (España) \\ rmartinez3196@gmail.com \\ Universidad Europea Miguel de Cervantes \\ Licenciada en Publicidad y Relaciones Públicas
}

\section{Resumen}

La Responsabilidad Social Corporativa (RSC) ○ Responsabilidad Social

Empresarial (RSE), es una práctica que surge como consecuencia de los cambios económico-sociales que se producen en la globalización y de la actuación

\section{Summary}

Corporate Social Responsibility (CSR) or Business Social Responsibility (BSR) is a practice that emerges as a consequence of economic and social changes that occur in globalization and in the global economy's performance. The company
RECIBIDO:

EvalUADO:

ACEPTADO:
19 DE AGOSTO DE 2015

12 DE NOVIEMBRE DE 2015

27 DE ENERO DE 2016
Para citar este artículo / to cite this article

Gómez Nieto, B. \& Martínez Domínguez, R. (2016). Responsabilidad Social Corporativa: de concepto abstracto a elemento clave en la estrategia empresarial, Poliantea, 12(22). 
de la economía a nivel mundial. La empresa no es un ente aislado, sino que forma parte de un entorno en el que debe estar perfectamente integrada como un elemento más del mismo. Lejos de ser una moda, la RSC en la actualidad se conforma como una práctica directamente vinculada con la actividad de las empresas y de las organizaciones que se valen del entorno, bien usando sus recursos (inputs) o abasteciéndolos (outputs). Todo ello está impregnado de unas demandas sociales que han permanecido latentes durante largos años. Esta realidad deriva en la necesidad de una concienciación social de todas las acciones empresariales que se desarrollan.

El objetivo de este estudio se centra en analizar la transformación y evolución del concepto de RSC hacia un elemento crucial de toda la estrategia de las organizaciones. Para ello se utiliza una metodología mixta: conceptual teórica y de análisis de contenido. La práctica de la RSC ha pasado de ser complemento de reconocimiento social a valor obligatorio en el ámbito empresarial y social.

Palabras clave: Responsabilidad Social Corporativa, globalización, empresa, relaciones públicas. is not an isolated entity, but part of an environment in which must be perfectly integrated as an additional element thereof. Far from being a fashion, the CSR today is intended as a practice directly linked with the activity of companies and organizations that rely on the environment, either by using its resources (inputs) or supplying them (outputs). All this is imbued with some social demands that have remained latent for many years. This reality leads to the need for social awareness of all business actions that are developed. The objective of this study focuses on analyzing the transformation and evolution of the CSR concept toward a crucial element of any organizational strategy. This is done using a mixed methodology: conceptual, theoretical and content analysis. The CSR practice has passed from being a social recognition complement to a required value in the social and business scope.

Key Words: Corporate Social Responsibility, globalization, enterprise, public relationships. 


\section{Introducción y estado de la cuestión}

La concepción más moderna de la empresa introduce nuevas formas de hacer negocio como la Responsabilidad Social Empresarial (RSC), considerando a la empresa como un ente social donde los grupos de interés se convierten en el objetivo principal de la estrategia corporativa; no se concibe empresa sin entorno (De la Cuesta, 2004).

En la actualidad, estamos insertos en una economía globalizada que deriva en un mercado, empresas, intereses, públicos... que no responden a los criterios locales o nacionales como ocurría hasta hace unas décadas, sino que responden a juicios de índole internacional (Bravo, Matute y Pina, 2011).

Arie de Geus ${ }^{1}$ señala que las compañías mueren porque sus

1 Autor de The Living Company. directivos se centran exclusivamente en producir bienes y servicios, $y$ se olvidan de que una empresa es una comunidad de seres humanos que está en el mundo de los negocios, cualquier negocio, para permanecer viva.

Las consecuencias medioambientales de las actividades comerciales de las empresas llevan poniendo en peligro la perdurabilidad del medioambiente tal y como lo conocemos. Y no solo eso, sino que se ha ido más allá. Ya no se exigen únicamente prácticas responsables de las compañías a nivel medioambiental, sino también social. Para Juan Romero, líder corporativo de Cemex, "ser líder en sustentabilidad es minimizar nuestra huella $y$ maximizar la contribución positiva a la sociedad, a la vez que fortalecemos nuestra competitividad a largo 
plazo, haciendo que el mundo sea un mejor lugar para vivir todos nosotros y las generaciones por venir" (Pizzolante Negrón, 2009, p. 249).

La significación de responsabilidad social corporativa (RSC) o empresa socialmente responsable (RSE), ha pasado de ser un concepto abstracto y mal entendido, a ser un apellido que otorga prestigio a las empresas que deciden adoptarlo. En pleno siglo XXI puede afirmarse con total firmeza que la RSC es un valor obligatorio que deben mostrar las empresas tanto en su ámbito externo como interno, debe ser un elemento que impregne su esencia y no un complemento que se limita al cumplimiento de unos estándares mínimos. Prueba de ello son todas las compañías que han hecho de un concepto novedoso una oportunidad de negocio (Abascal, 2005, p. 135).

La relevancia de este artículo se pone de manifiesto en la presentación de aspectos relevantes de la RSC, tales como concepto, orígenes, principios, valores, su implantación en la empresa y el tratamiento de la RSC como estrategia por medio de cuatro esferas bien diferenciadas y claves para comprender su esencia para la empresa actual. Se ha abordado el trabajo mediante una metodología conceptual-teórica y de análisis de contenido, para llegar a la conclusión de que en el entorno actual la RSC debe formar parte como valor intrínseco de las organizaciones y estas deben adoptarlo como tal si desean tener éxito en el mundo globalizado del que forman parte. La organización es, por lo tanto, un ser vivo que forma parte de esa comunidad interactuando con ella a distintos niveles.

\section{Material y métodos}

En este estudio se ha aplicado una metodología mixta: conceptual/ teórica y análisis de contenido. En primer lugar se desarrolla una metodología conceptual, puesto que se ha revisado en profundidad bibliografía, artículos científicos, estudios, datos sobre el concepto de RSC desde sus orígenes, y de análisis de contenido, puesto que se han analizado informes, estudios de expertos del ámbito nacional e internacional para determinar la evolución experimentada por la RSC desde varios enfoques: económico, social, cultural. Este planteamiento explica el uso de un análisis de contenido (Van Dijk, 2003, p. 149), que identifique la elección de temas, 
proposiciones, para el estudio de la RSC como elemento clave en la estrategia de las organizaciones.

\section{Sobre el concepto de responsabilidad social corporativa}

Según Lantos (2001), la proliferación de teorías, perspectivas y terminologías en la literatura académica que estudia los fenómenos asociados a la RSC ha provocado una elevada confusión cuando se pretende abordar su estudio.

Por lo tanto, existen varios marcos referenciales útiles para explicar el concepto y sus dimensiones (Alvarado, 2008). En efecto, entre los desarrollos académicos más destacables se hallan modelos tales como el de las Asociaciones Corporativas (Brown y Dacin, 1997); los basados en la teoría del Desarrollo Sostenible (Gladwin, Kennelly y Krause, 1995; Panapanaan, Linnanen, Karvonen y Phan, 2003; Panwar, Rinne, Hansen y Juslin, 2006; Wheeler, Colbert y Freeman, 2003); el de Zahra y Latour (1987), y la pirámide de Carroll (1991 y 1999), entre otros; y si bien estos coinciden, prácticamente en su totalidad, en señalar que la RSC es un constructo multidimensional, difieren entre ellos de forma importante, tanto en el número de dimensiones consideradas como en los propios factores que lo componen, dando como resultado que las aplicaciones desarrolladas en el ámbito profesional, como las identificadas en su oportunidad por Mercado y García (2007), resulten variopintas.

Se habla indistintamente de "responsabilidad social empresarial", "responsabilidad social corporativa" o "responsabilidad empresarial". $\mathrm{Y}$ además se confunde con otros términos como desarrollo sostenible, sostenibilidad y similares. En este caso se toma como referencia la definición aportada por la Comisión Europea en el Libro verde en 2001, que define la responsabilidad corporativa como "la integración voluntaria, por parte de las empresas, de las preocupaciones sociales $y$ medioambientales en sus operaciones comerciales y sus relaciones con sus interlocutores". Se concibe como "una contribución empresarial al desarrollo sostenible". En líneas generales, puede afirmarse que la responsabilidad corporativa se ha convertido en algo más que cumplir o acatar las exigencias legales que se estipulan en este campo, sino ir más allá integrando la RSC en los valores, 
gobierno, estrategia y operaciones en general de las empresas. Este concepto va más allá del comportamiento ilegal y antisocial que puedan ejercer las organizaciones, sea del tipo que sean (Sethi, 1975, p. 58).

El cambio en la concepción de empresa es doble. Por un lado se apela a los motivos éticos, pues la empresa es la institución social con mayor capacidad de innovación y contribución a un cambio positivo en la actual situación económica, social y medioambiental de nuestro planeta. Y por otro, una empresa se crea con el fin de que perdure el máximo de tiempo posible, por lo que la contribución de la empresa a la sostenibilidad del entorno en el que se desarrolla y compite se convierte en un elemento fundamental de su razón de ser. Desde este enfoque integrador, se asume que las empresas no solo cumplen sus obligaciones económicas, legales, éticas y sociales con respecto a sus accionistas, sino también hacia los empleados, clientes, comunidades locales, medioambiente, proveedores y distribuidores (Lafuente et al., 2003). Tomando como base esta perspectiva, la RSC se puede definir como "el reconocimiento e integración en sus operaciones por parte de las empresas, de las preocupaciones sociales y medioambientales, dando lugar a prácticas empresariales que satisfagan dichas preocupaciones $y$ configuren sus relaciones con sus interlocutores" (De la Cuesta y Valor, 2003, p. 11).

Boatright (2000, p. 23), define la responsabilidad social "como la adopción voluntaria de responsabilidades que van más allá de las responsabilidades meramente económicas y legales de la empresa". Por su parte, Archie B. Carroll (1999), señala que "la responsabilidad social empresarial abarca las expectativas económica, legal, ética y discrecional que la sociedad tiene de las organizaciones en un momento dado" (Aldeanueva, 2012, p. 10).

Contemplar la responsabilidad social corporativa como parte de la estrategia de las empresas pone de manifiesto la importancia de que esta sea conocida por todos los grupos de interés. Esta práctica no solo deben desarrollarla empresas de ámbito privado, sino también de índole gubernamental. Estudios como los de Escamilla et al. (2015), sobre la divulgación de la RSC en las empresas de trasporte público en España, por ejemplo, ponen de manifiesto que la información y el nivel de implantación de esta práctica son 
escasos. Otros estudios como los de Navarro et al. (2010), sobre la divulgación de la información de la RSC en las administraciones públicas de índole local, revelan que la información social es la más divulgada y la medioambiental la menos. Estos ejemplos muestran el interés por la transparencia de la información que se quiere presentar a los ciudadanos.

\section{Los orígenes de la responsabilidad social corporativa}

Para poder entender de dónde surgen las primeras cuestiones sobre responsabilidad empresarial se debe retroceder a los modelos económicos del siglo XX. Aquí se diferencian dos posturas totalmente contrarias. Por un lado, el pensamiento liberal liderado por Milton Friedman, quien afirma que la empresa solo tiene compromisos con la ley; y por otro lado, ideas keynesianas lideradas por Galbraith, quien asegura que además de atender a los compromisos legales debe tener en consideración los intereses de sus empleados y los del conjunto de la sociedad. Estas dos corrientes de pensamiento dan lugar a tres planteamientos (Polo, 2011, p. 34):
- La empresa solo tiene responsabilidades legales (liberales).

- Se acepta que la empresa tiene responsabilidades sociales y decide adoptarla de una manera voluntaria (la mayoría de las empresas).

- Es necesario implantar una normativa más estricta que regule estas responsabilidades.

Friedman expone sus argumentos en su artículo La responsabilidad social de las empresas es crecer sus ganancias, publicado en The New York Times. Está catalogado como uno de los ejes del pensamiento que pone en relación a la empresa y la sociedad. En él explica que las empresas no tienen responsabilidades porque son un sujeto abstracto, son los individuos que trabajan en ellas quienes tienen esas responsabilidades. Y añade que si los directivos se dejasen llevar por estos principios de responsabilidad social, lo que harían sería gastar, lo que implicaría que para que no se reduzca el dinero de los accionistas se tendría que subir el precio de los productos y servicios y el salario de los empleados se reduciría.

En contraposición a Friedman, de acuerdo con Polo (2011, p. 37), se 
encuentra lo que se considera como pensamientos keynesianos representados por:

- Frederick expone que sí deben responder las empresas a la responsabilidad social, ya que así lo ha determinado la opinión pública y las regulaciones del gobierno. $\mathrm{Y}$ expone que existen dos clases de RSC: RSC1: concepto puro de RSC, y RSC2: sensibilidad y capacidad para dar respuesta a las necesidades sociales.

- Sethi, para solventar las críticas sobre la dificultad de poner en práctica las propuestas de Frederick, distingue tres niveles: cumplir con las leyes, aceptar los valores sociales y mostrar sensibilidad ante los problemas del entorno.

- Archie Carroll va más allá, asegurando que las responsabilidades de la empresa deben dar respuesta a las aspiraciones legales, éticas y voluntarias que la propia sociedad espera de ella. De modo que plantea cuatro tipos de obligaciones: económicas (siendo esta la base), legales, éticas y voluntarias.
- Donna Wood amplía lo expuesto por Carroll, afirmando que sus niveles describen las complejas relaciones entre la sociedad, la empresa y los miembros de las organizaciones. Este cruce de intereses de cada uno de los niveles se puede clasificar en tres estratos: institucional: las empresas desarrollan sus actividades de manera responsable y dentro de la legalidad; corporativo: se aplican los criterios de responsabilidad pública y responsabilidad; individuales: los directivos como agentes morales.

Posteriormente a estas aportaciones se llegó a la instauración del criterio de los stakeholders, que se basa en la gestión de la empresa de acuerdo con sus públicos de interés. De esta manera, todas las decisiones emanadas de las instituciones estarán condicionadas por las necesidades, demandas y expectativas de esos públicos de interés.

Robert Phillips puntualiza que "la teoría de los stakeholders ${ }^{2}$ es

2 Stakeholders: aquellos grupos que pueden afectar o ser afectados por el logro de los propósitos de la organización. Obtenido el 28 de abril de 2013, de http://wnw.iese. 
distinta porque sitúa explícitamente moralyvalores como un rasgocentral de la gestión de las organizaciones".

En líneas generales, este modelo de stakeholders introduce una clave fundamental que es el diálogo que se mantiene con los diferentes públicos de la empresa, que combinado con las teorías del desarrollo sostenible produce el concepto de RSC moderno. Michael Porter y Mark Kranner exponen que hay cuatro motivos por los cuales apostar por la RSC: obligación moral, sostenibilidad, permiso para operar y reputación.

El punto de partida que justifica el estudio de la RSC es que los problemas medioambientales, sociales y económicos están interrelacionados.

El consumo de recursos es muy alto, se consumen más materias de las que la naturaleza puede ofrecer $y$, por tanto, regenerar. Una consecuencia directa de esta situación se plasma en el trabajo desarrollado por el World Resources Institute ${ }^{3}$, que en el 2005 evaluó el estado de los ecosistemas de

edu/es/files/La\%20evaluaci\%C3\%B3n\%20 del\%20concepto\%20de\%20stakeholders\%20seg\%C3\%BAn\%2OFreeman_tcm539688.pdf

3 Millenium Ecosystem Assessment. Ecosystems and Human Well-being: Synthesis. Island Press, Washington, D.C., 2005. la tierra poniendo en relación los tres elementos tratados: medioambiente, sociedad y economía.

\section{Principios de la RSC en una empresa}

Los fundamentos sobre los que se asienta el nuevo contexto de la empresa responsable y sostenible, de acuerdo a Olcese, Rodríguez y Alfaro (2008, p. 27), son: Apertura y sensibilidad hacia el entorno: apertura entendida como diálogo con los diferentes públicos que tiene la empresa. La capacidad de abrirse y dialogar proporciona a la empresa una visión sistemática que le facilita contemplar y entender la realidad e interactuar con ella, por un lado, y un conocimiento profundo de las necesidades y requerimientos del entorno y de las personas y organizaciones que lo representan, por otro. Estos elementos proporcionan más flexibilidad y capacidad de prever y adaptarse a los cambios, llegando a ser quien los promueva y lidere.

El sentido de comunidad se refiere a todas las personas que trabajan en una empresa y que forman parte de un todo con el que se identifican. Este sentido de comunidad se construye con base en dos pilares: 
1. una visión compartida: una de las disciplinas de una organización inteligente, es lo que une a sus integrantes en torno a un proyecto común y promueve que estos estén en condiciones y predispuestos a aportar todo su saber, potencial y experiencia.

2. unos valores compartidos: principios intrínsecamente valiosos y deseables. Para que haya armonía, los valores de la empresa y los de las personas que la forman han de ser coherentes, ya que actúan como la conciencia de la organización.

Los activos intangibles representan más del $85 \%$ del valor de las empresas y son los que permiten desarrollar a las empresas ventajas competitivas con respecto a su competencia. Se caracterizan por tener recursos y capacidades que los tildan de raros (infrecuentes), difíciles de imitar y no comercializables. El conocimiento es uno de estos activos intangibles que se estimula mediante las interrelaciones de las personas que forman los equipos.

Se define como activo intangible complejo a los recursos basados en el establecimiento de relaciones con los grupos de interés, interno y externos, que son capaces de generar nuevos conocimientos, sirven de base a la innovación radical y permiten el desarrollo y mantenimiento de ventajas competitivas sostenibles que no son medibles con parámetros contables o físicos.

Para Hart y Sharma (2004, p. 1), las innovaciones más radicales provienen del propio sistema, por eso es enriquecedor mantener lazos no solo con los públicos a lo que afecta directamente la organización.

\section{Valores que consolidan la RSC}

Para que los miembros de una compañía actúen y tomen decisiones de la forma más adecuada para la empresa en su conjunto, es imprescindible la existencia de una fuerte cultura basada en la interiorización de unos valores compartidos.

La gran importancia que ha ido ganando la empresa como institución ha derivado en que los valores empresariales tengan una tremenda influencia en los valores sociales. Mintzberg (2002, p. 124) presenta cinco asunciones que la práctica empresarial y la teoría económica han conseguido que se conviertan 
en verdades incuestionables, que han afectado profundamente a la evolución social:

1. Todos somos "homo economicus": cada uno está preocupado por su propio interés y se busca de manera racional y calculadora maximizar las propias ganancias.

2. Las empresas existen para maximizar el valor de los accionistas, por lo que las necesidades y méritos del resto están supeditadas a la consecución de este fin.

3. Las corporaciones requieren líderes heroicos: el líder (el ejecutivo) es la corporación y es el único responsable del rendimiento de toda la corporación.

4. La organización eficaz es magra: despedir a trabajadores solo tiene ventajas.

5. Una pleamar de prosperidad hace que todos los barcos floten: la economía basada en el egoísmo da como resultado que afecte positivamente a toda la sociedad en su conjunto y hace que todos prosperen.

Estas cinco afirmaciones han promovido la desconfianza entre las personas, la ruptura entre propietarios y empleados, la desconexión entre presente y futuro, $y$ la creciente disparidad entre ricos y pobres.

Los valores compartidos son importantes desde una doble óptica: por un lado, por la trascendencia que tienen en la forma de actuar y decidir desde las empresas; $y$ por otro, por la influencia que tienen en el conjunto de la sociedad, por lo que la empresa sostenible debe implicar una serie de valores positivos y orientados al crecimiento global y grupal.

Los valores que reinaban en la empresa tradicional son: bien propio, la competitividad, la imagen, ser racional, la jerarquía, la uniformidad, la comunicación, el control y la eficiencia, ya que todos estos elementos se conjugaban para crear valor para la empresa.

Por su parte, la empresa sostenible ha de ser capaz de conjugar los antiguos valores con los nuevos, para que se pueda desarrollar la compañía sobre los cinco fundamentos explicados anteriormente (Olcese, Rodríguez y Alfaro, 2008, pp. 38-46). 


\section{La estrategia de la Responsabilidad Social Corporativa}

\section{RSC como estrategia de sustentabilidad}

El adecuado programa de Responsabilidad Social debe estar alineado con el plan de negocios de la empresa, lo que deriva en una estabilidad a lo largo del tiempo que no depende únicamente de los éxitos logrados en el campo económico. El punto de inflexión consiste en dotar de poder a los profesionales que gestionan esta responsabilidad social de la empresa, independientemente del puesto jerárquico que ocupen dentro de la organización. Así podrán dirigir cada una de las acciones que desarrolle la compañía. Para Negrón (2009, p. 219), con esto se consigue que estos empleados puedan defender sus ideas delante del alto mando de la empresa, sean un voto más a tener en cuenta en el diseño estratégico de las actuaciones y se convier$\tan$ en el puente fundamental entre la alta esfera de la empresa y los trabajadores de los mismos.

Según el Modelo de la Fundación de la Escuela de Gerencia Social (FEGS), este concepto de responsabilidad social está fundamentado en cuatro pilares ${ }^{4}$ :

- Eliminación de las distancias entre la acción social del Estado y de las empresas, ya que el Estado no es capaz de solventar los conflictos sociales por sí mismo.

- Nueva conciencia empresarial determinada por implicarse directamente con la comunidad resolviendo problemas sociales que puedan surgir tanto a nivel interno como externo, respecto de la organización.

- Incorporar mejoras que repercutan en los trabajadores.

- Alcanzar las expectativas que la sociedad tiene sobre la empresa, otorgando una nueva legitimidad a la acción empresarial.

De acuerdo con García-Marzá (2004, p. 54), este poderoso capital empresarial que trata de expectativas de reciprocidad, de valores y normas compartidas, de canales informales de cooperación y de información...

4 Fundación Escuela de Gerencia Social, Ministerio de Planificación y Desarrollo, Venamcham. Balance social para la empresa venezolana. Modelo de la Fundación Escuela Gerencia Social FEGS, Caracas, 2002 (p. 18). 
que constituyen el entramado social más importante y básico, sobre el que después se construirán los mecanismos formales tales como el derecho o la empresa como institución social así como toda su actuación estratégica.

\section{La implantación de la \\ RSC en la empresa}

Es fundamental partir de la base de que la comunicación estratégica es el vehículo clave para que, junto a una coherente y clara estrategia de actuación social y mensaje, consiga construir perspectivas que faciliten la consecución de los objetivos planteados.

El objetivo para Negrón (2004, p. 290) es alcanzar un modelo de actuación responsable que permita alcanzar la misión y visión de la empresa, modo que debe interiorizarse como una pieza estructural más de la cultura del trabajo. Esta realidad permite tener una visión compartida, fortalecer institucionalmente a la empresa generando más coherencia y consistencia en el trabajo, proyectando más credibilidad en el seno interno y externo de la empresa. Este nuevo logro se puede definir como una herramienta gerencial para la construcción de la reputación corporativa, que permite el fortalecimiento institucional de las empresas socialmente responsables, construyendo confianza con sus públicos internos y externos y armonizando sus intereses con las expectativas de cada uno de ellos (Negrón, 2009, p. 294). Este modelo está integrado por tres esferas: estrategia, mensajes y habilidades, todas envueltas en un cuarto pilar denominado governance. Todos ellos conviven bajo la premisa de que tanto el entorno como el dintorno condicionan la actividad de la empresa y el logro de sus objetivos.

A continuación presentamos un cuadro en el que se refleja el esquema que se va a desarrollar ${ }^{5}$ :

5 Obtenido el 26 de julio de 2013, de http://www.fenalco.com.co/contenido/1891 
Cuadro 1 : Esquema de las esferas que conforman la RSC como estrategia de sustentabilidad.

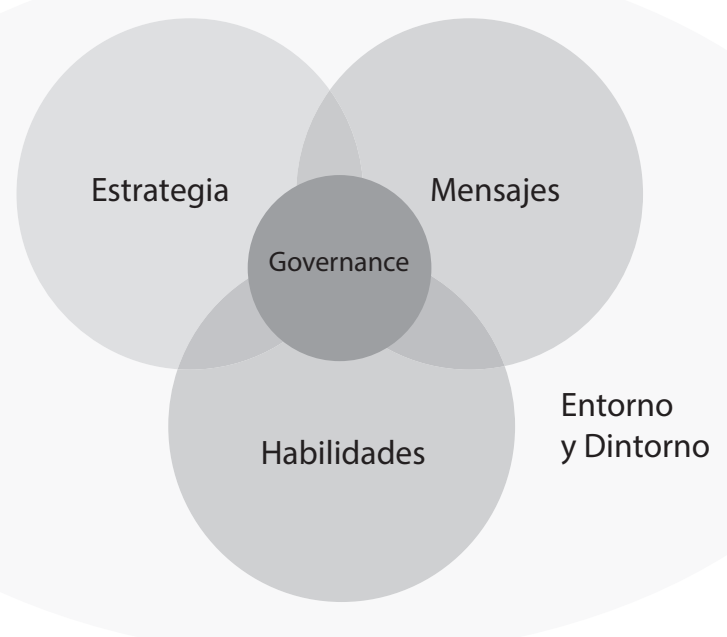

Fuente: http://www.fenalco.com.co/contenido/1891

\section{La primera esfera: la Estrategia}

La acción social debe estar incluida Empresa Socialmente Responsable, en la estructura base de la planifi- sino que esa visión debe estar articación de la compañía, pues no se culada con la definición formal de debe olvidar el hecho de que la RSC los mensajes que comunica, las haes un valor sostenible en el tiempo bilidades que requiere para lograr porque debe ser capaz de ajustar la sustentabilidad de su actuación los intereses de la empresa con las y un modelo de negocio que sepa expectativas del entorno en el que se desarrolla. El capital humano es un factor clave a tener en cuenta en todo el entramado de la RSC, y en ocasiones surge por iniciativa propia de la persona. Por ello es necesario un modelo que no solo se centre en un pensamiento estratégico sobre el rol que le corresponde a una construir la empresa para que cada una de las dimensiones esté orientada al logro.

Con el fin de facilitar este trabajo, Pizzolante (2009, p. 319) propone una herramienta llamada mapa de actuación estratégica. Estos mapas son una representación gráfica interconectada de instancias, 
organizaciones, grupos e individuos cuyos contactos, relaciones e influencias son determinantes en la toma de decisiones por parte de los gobiernos, poderes del Estado u otros grupos de presión que impactan en el Plan de Negocios de la empresa. Esta matriz está compuesta por cuatro columnas que permiten volcar toda la información relevante de la organización en ellas, facilitando la comprensión actual de la misma. Son cuatro columnas: macro-mensajes, voceros, medios y públicos.

- Macro-mensajes. Son líneas gruesas de los mensajes que la empresa debería comunicar con las diversas audiencias.

- Voceros. Se refiere a determinar todas las personas que podrían desarrollar esta actividad en cualquiera que sea la situación que se produzca, independientemente de su jerarquía dentro de la organización.

- Medios. Se trata de definir todos los medios de comunicación disponibles.

- Públicos. Se refiere a que los externos deben estar bien identificados y definidos, y el interno bien informado.
Gracias a esta herramienta se dispone de una matriz que refleja las diferentes acciones contenidas en el Plan de Trabajo. Además, esta herramienta ha sido mejorada y completada posteriormente, como es el caso de los públicos, los cuales en función de su grado de influencia se dividen en:

- Prescriptores. Su opinión es considerada como concluyente.

- Mediadores. Construyen puentes entre personas de diferentes opiniones y tienden a armonizar los diversos intereses de todos los implicados.

- Neutros. No hacen juicio de valor.

- Detractores. Es el grupo más completo, porque cuando emiten su opinión siempre es una crítica que deriva en censura y cuestionamiento de la empresa.

Otra herramienta que destaca Negrón (2009, p. 323), en este modelo de actuación responsable, son las Guías de actuación responsable. Se define como el instrumento de gestión que le asegura a la empresa socialmente responsable aquello que debe hacer, porque determina cuántas oportunidades de comunicación 
se pueden aprovechar en el proceso de lograr un objetivo y asignarle a cada una de esas oportunidades el mensaje y medio más adecuado.

\section{La segunda esfera: los Mensajes}

Los mensajes son una pieza fundamental para la formación de una guía de mensajes fuerte y sostenible en el tiempo, porque son una herramienta sencilla de gran uso para la divulgación interna, que permite la homogeneización en cuanto a contenido sobre el tema entre todos los empleados y un ajuste coherente entre lo que hace y comunica. Dichos mensajes deben estar estructurados y alineados a la estrategia definida previamente. Una visión sustentable solo se produce cuando se cuenta con mensajes claros y concretos que se materializan en la forma de ser y hacer de la compañía. Para ello se debe atender tanto a los intereses del entorno como a los del dintorno. Los mensajes deben permitir comprender el estilo de la empresa, sus intenciones, su misión y visión y la coherencia de sus mensajes con lo que hace realmente (Negrón, 2009, p. 327).

El fin es obtener mensajes compartidos con los diferentes grados jerárquicos de la empresa y con los públicos internos y externos, para establecer posteriormente los Ejes de Posicionamiento Informativo de la institución.

\section{La tercera esfera: Ias Habilidades}

Para diseñar un programa de desarrollo de habilidades que asegure que la actuación del recurso humano de la organización comunique los atributos que caracterizan a la empresa socialmente responsable, es necesario que haya una institucionalidad liderada por el equipo gerencial de la empresa. Una de las claves es conectar los mensajes verbales con los mensajes no verbales propios de la actuación, pues la confianza que se tiene en un mensaje se basa un $90 \%$ en percepciones que construye la empresa mediante las formas a través de las cuales se comunican aquellos. Es fundamental, por lo tanto, transmitir con efectividad los mensajes verbales en equilibrio estratégico con nuevas capacidades no-verbales (Negrón, 2009, p. 336).

Son las habilidades desarrolladas por el capital humano que conforman la empresa, las que permiten evolucionar de la tradicional RSC hacia la idea clave: crear una conciencia 
colectiva respecto a la RSC compartida por todo el capital humano de la organización, y no solo por aquellos que se dedican en exclusiva a ello, de manera que la empresa se encuentra en un proceso continuo.

Cuando la dimensión de los mensajes se cruza con la dimensión de las habilidades es imprescindible comprender que la palabra puede comunicar diferentes mensajes y grandes contradicciones que atentan contra lo que realmente se desea comunicar.

Una empresa socialmente responsable debe destinar muchos esfuerzos a evaluar permanentemente el diálogo que la empresa sostiene con sus diferentes públicos y aprender de ellos.

Por otra parte, cuando la dimensión de mensajes se cruza con la dimensión de las habilidades, es necesario recordar que las capacidades organizacionales que caracterizan a los líderes que conducen las empresas deben estar directamente en armonía con los objetivos explícitos de la empresa y el cumplimiento de su plan de negocios.

\section{La cuarta esfera: Governance}

El concepto de "Governance" hace referencia al modelo de gobierno.
Hace hincapié en la forma particular de cómo nos gestionamos, es decir, si somos responsables o no frente a la comunidad de empleados y a la sociedad en la que nos desarrollamos. Por governance se entiende la manera en que se organiza, estructura interna y externamente la empresa y establece relaciones con sus stakeholders (Negrón, 2009, p. 342).

Debe haber un área exclusiva para el manejo de la información de RSC, aparte de ser una constante en todos los ámbitos de la empresa. Pero esto no significa que esta área sea la única que deba gestionar actividades para consolidar la credibilidad y reputación de la empresa frente a la opinión pública.

Es importante tener en cuenta la dimensión governance, porque la actitud de las empresas no está siempre en concordancia con la gestión que hacen de sus actividades, y esta doble visión es lo que perjudica a la credibilidad y a los proyectos de RSC. El cometido de esta dimensión consiste en desarrollar instancias gerenciales necesarias para estimular la aparición del pensamiento estratégico dentro de los equipos profesionales de la alta dirección que conforman la empresa, así como desarrollar una metodología que 
permite equilibrar las visiones entre el liderazgo y el capital humano del que se dispone para ser sustentables a lo largo del tiempo. Esto desembocará en un logro de los objetivos definidos en el plan de negocios.

La combinación de las tres esferas: estrategia, mensajes y habilidades, todas ellas dirigidas bajo la governance, da lugar a un modelo de responsabilidad social empresarial que goza de una licencia social para operar en la sociedad (Negrón, 2009, p. 349).

Además, para poner en práctica una adecuada y sólida política de RSC son necesarias las siguientes herramientas (Polo, 2011, p. 64):

- Declaraciones corporativas: comunicar los valores y principios de la empresa (misión y visión).

- Códigos de conducta: reflejar por escrito los códigos morales a los que su capital humano debe ceñirse.

- Políticas de RSC: lideradas por la alta esfera de la empresa que debe impregnar a todas las ramificaciones de la compañía.

- Sistemas de gestión: pautas de actuación.

- Informes sociales y medioambientales: la más reconocida GRI
(Global Reporting Iniciative), que lucha por establecer cánones comunes a nivel internacional. Y la inversión socialmente responsable consecuencia de los proyectos de RSC evaluada por DJSI y FTSE4Good.

Otra manera de introducir la RSC en la gestión estratégica de la empresa es mediante el Balance Scorecard6. El BSC es una herramienta que destaca por su sencillez en la gestión de intangibles de las empresas, por ello incluir un proceso tan complejo como la responsabilidad social corporativa en ella, supone una manera sencilla para su tratamiento y adaptación. Aunque lo ideal sería crear un BSC propio y único para la RSC.

En el caso de que no se diseñe un BSC específico para la RSC es complejo ubicar la posición que esta ocuparía en el engranaje. Al considerarse un proceso interno, se ubicaría entre los procesos internos y el aprendizaje y crecimiento de la

6 El BSC (Balanced Scorecard) es una herramienta metodológica que traduce la estrategia en un conjunto de medidas de actuación, las cuales proporcionan la estructura necesaria para un sistema de gestión y medición. 
empresa, pues se requieren la definición de estándares de actuación y la puesta en marcha del sistema de gestión medioambiental. La utilización de esta herramienta es práctica, porque facilita la gestión de los cambios al transformar la estrategia en objetivos operativos, y en concreto en la RSC ayuda a la alineación de objetivos y comportamiento del capital humano, reformulación de la estrategia, transformación de actuaciones de largo plazo en corto plazo y facilitar la creación de valor futuro (López y Llena, 2006).

Es fundamental que las empresas adopten todos los aspectos que contempla la responsabilidad social corporativa para obtener ventajas competitivas en el mercado, lo que se traduce en introducir la RSC en el sistema de gestión y, sobre todo, en la gestión estratégica.

\section{Conclusiones}

La responsabilidad social de la empresa alcanzó gran relevancia hace varias décadas. La RSC ha fortalecido y expandido algunos de los principios de entonces. Las numerosas iniciativas mundiales y regionales ponen de manifiesto la importancia de la triple perspectiva de la RSC, económica, social y medioambiental (Bigné et al., 2005).

En la actualidad, incluir la RSC en la estrategia integral de la empresa (objetivos y cultura empresarial) es clave fundamental para su éxito. Una compañía consigue ser buena en RSC si cumple con los factores (Vargas Sánchez, 2009):

- Compromiso de la alta dirección y propietarios.

- Adopción de los valores y la visión de la RSC en la organización y su cultura.

- Implementación de la RSC en la estrategia, procesos de gestión y las políticas, y en las prácticas diarias y sistemas futuros.

- Fijar objetivos, diseñar planes para su consecución y desarrollar métodos para su evaluación y comunicación.

- Comunicación transparente, directa y con un lenguaje adaptado a los públicos de la estrategia, objetivos o actividades para motivar la participación de los implicados.

- Apertura para aprender, innovar y mejorar.

- Trabajar con terceros atendiendo a cómo desarrollan sus proyectos para aprender de ellos y 
plantear la posibilidad de desarrollar actividades y acuerdos conjuntos.

- Hacer de todos los valores, conceptos y todo lo relacionado con la RSC el capital humano de la empresa.

- Compartir experiencias, en redes, foros, ejemplos de buenas prácticas, benchmarking, etc.

Plantearse la RSC como estrategia de sustentabilidad implica que la RSC no es un asunto a tratar como un departamento más dentro de la empresa, sino que debe interiorizarse en la esencia de la empresa y aplicarse en cada una de las ramificaciones de la misma. Debe estar alineado con el plan de negocios de la empresa para que el éxito no dependa únicamente de los éxitos logrados en el campo económico. Esto implica la conexión de dos piezas: la empresa que asume su responsabilidad con su país y ciudadanía en las que opera, y las comunidades deben participar en este conglomerado económico-social.

La responsabilidad social empresarial es una realidad que está formada por tres vértices, $\mathrm{y}$ es la conjugación de todos ellos lo que da lugar a una buena estrategia de sustentabilidad para seguir por una empresa que pretende alcanzar el éxito el día de mañana: retos económicos, sociales y medioambientales.

La correcta combinación de estas tres claves debe integrarse en la estrategia de la empresa con visión a largo plazo, para poder adecuar las actuaciones a los cambios que se producen en el entorno, dependiendo del proceso a realizar de cada momento. La comunicación estratégica es el vehículo clave para que junto a una coherente y clara actuación social y en el mensaje, se consiga construir planes que faciliten su consecución.

$\mathrm{El}$ asentamiento de los valores compartidos por todos los miembros de la organización es fundamental por la trascendencia que tienen en la forma de actuar y por la influencia que tienen en el conjunto de la sociedad. De este modo, la RSC ayuda en la construcción de una empresa sostenible orientada al crecimiento global y grupal. 


\section{Referencias}

Abascal Rojas, F. (2005). Marketing social y ética empresarial. Madrid, España: ESIC.

Aldeanueva, I. (2012). La responsabilidad social como elemento de cohesión en la integración de los sistemas de gestión. Revista de Dirección y Administración de Empresas, (19), 9-28.

Alvarado, A. y Schlesinger, M. W. (2008). Dimensionalidad de la Responsabilidad Social Empresarial percibida y sus efectos sobre la imagen y la reputación: una aproximación desde el modelo de Carroll. Estudios gerenciales, 24(108). Recuperado de http://www.scielo.org.co/scielo.php?pid=S0123$59232008000300002 \& \mathrm{script}=$ sci_arttext.

Bigné, E.; Andreu, L.; Chumpitaz, R. y Swaen, V. (2005). Percepción de la responsabilidad social corporativa: un análisis cross-cultural. Universia Business Review, (5), 14 - 27.

Boatright, J. R. (2000). Ethics and the Conduct of Business. Upper Saddle River, NJ: Prentice Hall.
Bravo, R.; Matute, J. y Pina, J. M. (2011). Corporate social responsibility as a vehicle to reveal the corporate identity: A study focused on the websites of Spanish financial entities. Journal of Business Ethics.

De la Cuesta, M. (2004). El por qué de la responsabilidad social corporativa. Boletín Económico ICE, (2813), 45-57.

De la Cuesta, M. y Valor, C. (2003). Responsabilidad social de la empresa. Concepto, medición y desarrollo en España. Boletín económico del ICE, (2755), 7-19.

De Geus, A. (1997). The living Company. Boston, U.S.A: Harvard Business School Press.

Fundación Escuela de Gerencia Social, Ministerio de Planificación y Desarrollo, Venamcham (2002). Balance social para la empresa venezolana. Modelo de la Fundación Escuela Gerencia Social FEGS, Caracas.

Escamilla, S.; Plaza, P. y Flores, S. (2015). Análisis de la divulgación de la información sobre responsabilidad social corporativa en las 
empresas de transporte público urbano en España. Revista de Contabilidad - Spanish Accounting Review.

García-Marzá, D. (2004). Ética empresarial del diálogo a la confianza. Madrid, España: Editorial Trotta.

Hart, S. L. y Sharma, S. (2004). Engaging Fringe Stakeholders for Competitive Imagination. Academy of Management Executive, 18(1). Recuperado de http://www.iese.edu/es/ files/La\%20evaluaci\%C3\%B3n\%20 del\%20concepto\%20de\%20stakeholders\%20seg\%C3\%BAn\%20Freeman_tcm5-39688.pdf

Lafuente, A.; Viñuales, V.; Pueyo, R. y Llaría, J. (2003). La responsabilidad social corporativa y políticas públicas. Madrid, España: Fundación Alternativas.

Lantos, G. P. (2001). The boundaries of strategic corporate social responsibility. Journal of Consumer Marketing, 18(7), 595-632.

López Viñegla, A. y Llena Macarulla, F. (2006). La incorporación de la responsabilidad social corporativa en la gestión estratégica a través del Balanced Scorecard. Recuperado de http://ciberconta.unizar.es/LECCION/medio3-12/BSC_RSC.pdf].
Millenium Ecosystem Assessment. (2005). Ecosystems and Human Well-being: Synthesis. Washington, D.C., U.S.A.: Island Press,

Mintzberg, H.; Simons, R. y Basu, K. (2002). Beyond Selfishness, MIT Sloan Management Review, Fall.

Navarro, A.; Alcaraz, F. J. y Ortiz, D. (2010). La divulgación de la información sobre responsabilidad corporativa en administraciones públicas: un estudio empírico en gobiernos locales. Revista de Contabilidad - Spanish Accounting Review, 13 (2), pp. 285 - 314.

Olcese, A.; Rodríguez, M. A. y Alfaro, J. (2008). Manual de la empresa responsable y sostenible. Madrid, España: Mc Graw Hill

Pizzolante Negrón, I. (2004). Gestión de la comunicación en las organizaciones. Barcelona, España: Ariel.

Pizzolante Negrón, I. (2009). De la responsabilidad social empresarial a la empresa socialmente responsable. Castellón, España: Ciencias Sociales. Polo Martín, J. F. (2011). La RSC de las grandes empresas españolas con presencia en América Latina. Madrid, España: Fragua. 
Sethi, S. P. (1975). Dimensions of Corporate Social Performance: An analytical framework. California Management Review, 17(3), 58-64.

Van Dijk, T. A. (2003). La multidisciplinaridad del análisis crítico del discurso: un alegato a favor de la diversidad, en R. Wodak \& M. Meyer (eds.), Métodos de análisis crítico del discurso. Barcelona, España: Gedisa. Vargas Sánchez, A. (2009). Responsabilidad Social Corporativa y Dirección de los $R R H H$, Universidad de Huelva. Recuperado de http:// www.observatoriorrhhturismo.org/ FilesDispatcher;jsessionid $=062 \mathrm{BD}$ E5FD145F3448FE277331B50B07C ?id=3661\&tipo $=$ PublicacionAnexo 